\title{
Progress of different treatment modalities to limit the use of antibiotics in the treatment of acne
}

\author{
Kiran Sanjel ${ }^{1,2}$, Xue Mei Zhang ${ }^{1,2}$
}

${ }^{1}$ Clinical Medical School of Inner Mongolia University for the Nationalities, Tongliao, Inner Mongolia 028000, China,

${ }^{2}$ Department of Dermatology and Venerology, the Affiliated Hospital of Inner Mongolia University for the Nationalities, Tongliao, Inner Mongolia 028000, China

Corresponding author: Kiran Sanjel, MD, E-mail: kiransanjel@gmail.com

\begin{abstract}
Acne vulgaris is one of the most common skin diseases, affecting mainly teenagers. Its treatment procedure is complex, with a long duration of medication. Antibiotics are the most preferably prescribed drugs for the treatment of acne. The long-term use of antibiotics leads to various adverse effects such as the disruption of indigenous flora and resistance. Therefore, numerous therapeutic protocols such as antimicrobial stewardship have been proposed to limit the haphazard use of oral antibiotics. This review emphasizes different topical drugs, systemic alternative drugs, laser, and light therapy, as effective therapies for acne. This review also briefly reflects the efficacy of fire needle therapy-a traditional Chinese therapy-for acne.
\end{abstract}

Key words: acne vulgaris; antibiotic resistance; fire needle therapy; isotretinoin; Clascoterone

\section{INTRODUCTION}

Acne vulgaris is a common chronic, multifaceted inflammatory disease of the pilosebaceous unit. Although acne may be self-limiting, the sequelae may be lifelong. Early diagnosis and treatment of acne is the modality to decrease acne sequelae. Oral antibiotics are a common choice as a drug for acne. Although different guidelines have been implicated, oral antibiotics are prescribed haphazardly for a very long time in acne vulgaris. Antibiotics resistance has been an alarming public health concern for the last three decades, including in dermatology. Resistance to topical erythromycin, clindamycin, and systemic tetracyclineclass antibiotics are increased in Cutibacterium acne (formerly Propionibacterium acne) [1]. Excessive oral use of antibiotics leads to the disruption of indigenous gut flora [2]. Besides that, upper respiratory tract infection and an increase in pharyngitis are also involved [3]. Longer antibiotic use might be associated with inflammatory bowel disease [4]. At the same time, there is an association between the use of oral tetracycline-class antibiotics and the risk of breast and colon cancer $[5,6]$. This article mainly emphasizes the different alternative treatment modalities for decreasing oral antibiotic use in acne vulgaris.

\section{Topical Drugs}

\section{Benzoyl Peroxide (BPO)}

$\mathrm{BPO}$ is one of the over-the-counter medications for acne vulgaris, with formulation concentrations of $2.5 \%$, $5 \%$, and $10 \%$. It is a powerful antimicrobial agent, inhibiting bacterial protein and nucleotide synthesis, metabolic pathways, and mitochondrial activity. It also acts as a mild sebostatic and keratolytic agent [7]. This mechanism allows benzoyl peroxide to be employed as a long-term therapy for acne. It is useful either as a monotherapy or in combination with topical retinoids or antibiotics, without the risk of developing bacterial resistance. It is a therapy for mild acne or as an adjunctive therapy for moderate to severe acne. It is available in lotions, creams, gels, foams, solutions, cleansing bars, masks, and shaving creams. A recent

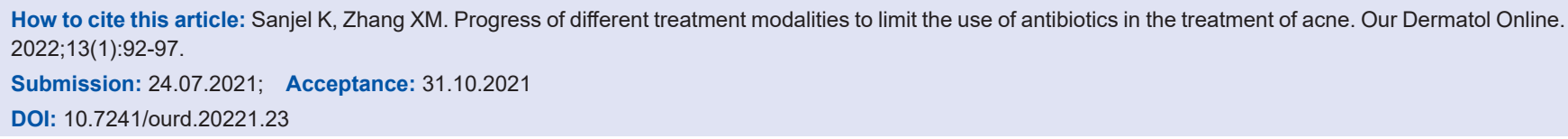


2020 Cochrane review on benzoyl peroxide assessed that BPO is more effective than placebo and is as effective as topical retinoids and topical antibiotics [8].

\section{Safety Level}

Bleaching of hair and color fabrics are the most common side effects, together with dryness, erythema, and scaling in higher concentrations. Some portion of the literature reports cases of contact dermatitis. It is better to begin with a low dose and concentration and gradually increase as the skin develops tolerance. FDA classifies benzoyl peroxide as pregnancy risk category $\mathrm{C}$. It is considered safe for breastfeeding until and unless benzoyl peroxide comes directly in contact with the area with which the infant could have direct contact during breastfeeding [9].

\section{Topical Retinoids}

First-line therapy for mild to moderate acne is topical retinoids. Retinoids activate the retinoic acid receptors, which affect the expression of genes involved in cell proliferation, differentiation, and inflammation. Thus, retinoids have both comedolytic and anti-inflammatory actions devoid of bacterial resistance. Tretinoin and tazarotene also suppress toll-like receptor expression, causing inflammatory cytokines and nitric oxide levels to decrease. Among topical retinoids, the most tolerated is $0.3 \%$ adapalene gel as it is photostable is the only topical retinoid that may be combined with $2.5 \%$ benzoyl peroxide without degradation; these are the most effective in moderate to severe acne [10]. A recent post hoc analysis showed that tretinoin $0.05 \%$ lotion was more effective in reducing non-inflammatory acne with no treatment-related adverse effects [11]. Recently, a fourth-generation topical retinoid-trifarotene-has been shown to have a selective retinoic acid receptor gamma agonist action. A three-phase randomized evaluation of 50 micrograms of trifarotene for facial and truncal acne for twelve weeks showed a reduction in the number of inflammatory lesions compared to the vehicle [12]. It has a better safety profile in comparison to other retinoids.

\section{Safety Level}

Topical retinoids can irritate more sensitive skin. Excessive use may result in redness, peeling, and blistering in the used area. Peeling of the stratum corneum may increase the chances of sunburn, so sunblock measures are needed. Adapalene and tretinoin are pregnancy category $\mathrm{C}$, whereas tazarotene is a category X drug [13]. Since retinoids are absorbed poorly after topical use, these are considered low-risk to nursing infants [13], ensuring that the skin is not in contact with the treated area.

\section{Topical Anti-Androgen}

Clascoterone is a new topical anti-androgen medication approved by the FDA in 2020 for the topical treatment of acne [14], which mainly targets testosterone and dihydrotestosterone [15]. It is available as $1 \%$ cream. It binds to the androgen receptor on the site of topical application. It is metabolized to an inactive form quickly, which limits its systemic absorption [15]. One of its advantages is that its onset of action is rapid, resulting in an improvement within two weeks. Both females and males older than twelve years are allowed to use it $[16,17]$. Two large phase three randomized controlled trials evaluated the effectiveness of clascoterone administered for twelve weeks, reporting a decrease in acne lesions and its symptoms by $8-18 \%$ more than placebo [18].

\section{Safety Level}

To date, the literature has reported no contraindications of this drug. The side effects include itching, burning, peeling, and dryness of the skin. Suppression of the hypothalamic-pituitary-adrenal axis is its severe side effect due to its cortexolone metabolites [13]. Hyperkalemia occurs in 5\% of clascoterone-treated individuals [15]. Ophthalmic, oral, or vaginal routes are not prescribed for use. No data regarding pregnancy and nursing women is available.

\section{Systemic Drugs}

\section{Oral Spironolactone}

Spironolactone-a synthetic 17-lactone steroid-is an aldosterone antagonist. It functions as an androgen receptor blocker and inhibitor of $5 \alpha$-reductase in the context of acne treatment. As it decreases sebum production, it is used for acne for more than thirty years [19]. In recent years, the use of spironolactone has increased considerably, but the use of oral antibiotics has not declined among females with acne [20]. Thereby, increased use of spironolactone might be an opportunity to improve antimicrobial stewardship. It is effective for acne in females of all ages. Spironolactone combined with a topical retinoid seems to provide a superior response to retinoid monotherapy 
in adult female acne. A recent retrospective review performed on eighty adolescent females showed an $80 \%$ improvement in acne lesions with few side effects [21]. A retrospective study done in 2017 stated that women treated with spironolactone at $100 \mathrm{mg} /$ day showed an improvement in $80 \%$, with only $4 \%$ experiencing any side effects [22]. FASCE and SAFA, two randomized trials, are being conducted to compare the efficacy of spironolactone over oral antibiotics. The results of these trials anticipated making the drug FDA approved [23]. Dosing is $25-200 \mathrm{mg} /$ day, starting from $100 \mathrm{mg} /$ day.

\section{Safety Level}

The side effects include diuresis, potential hyperkalemia, irregular menstrual bleeding, breast tenderness, headache, and fatigue. Combining spironolactone with oral contraceptives may decrease the problem of irregular menstrual bleeding. In healthy young females without cardiovascular disease, hypertension, or renal disease and not taking any interacting medicine, there is no evidence of increased potassium levels [24]. The contraindications include renal impairment, hyperkalemia, taking medication that increases serum potassium, and Addison's disease. Spironolactone is pregnancy category $\mathrm{C}$. Due to the evidence of teratogenicity in animal studies with high doses, it received a black box warning from the FDA [25]. Regarding breastfeeding, the risk to the infant is minimal.

\section{Oral Contraceptives}

Combined oral contraceptives focus on four mechanisms of the hormonal pathogenesis of acne:

1. They decrease the amount of gonadal androgen production;

2. They decrease 40-50\% of free testosterone [26];

3. Estrogen reduces the conversion of testosterone to dihydrotestosterone in the pilosebaceous unit, decreasing sebum;

4. Progestins with an anti-androgenic effect block the androgen receptors on keratinocytes and sebocytes.

It is effective in the treatment of both non-inflammatory and inflammatory acne. Ethinylestradiol + cyproterone acetate shows efficacy comparable to minocycline and superior efficacy compared to tetracycline [27]. A Cochrane review emphasized the effectiveness of all oral contraceptives for the treatment of acne in females. In trials with drospirenone-containing combined oral contraceptives compared with other oral contraceptives, the former have been generally favored [28]. A course of 3-6 months of therapy is required. The three oral contraceptives approved by the FDA for the treatment of acne are norgestimate (180 mg, $215 \mathrm{mg}, 250 \mathrm{mg})+$ ethinylestradiol ( $35 \mathrm{mcg}$ ), ethinylestradiol (20-35 mcg) + norethindrone acetate $(1 \mathrm{mg})$, and ethinylestradiol $(20 \mathrm{mcg})+$ drospirenone $(3 \mathrm{mg})$.

\section{Safety Level}

The common side effects include breakthrough bleeding, nausea, breast tenderness, and increased risk of thromboembolic events (attributable risk). While counseling, it is emphasized that the risk of venous thromboembolism is higher in pregnancy than in combined oral contraceptive use [29]. It is pregnancy category $\mathrm{X}$ and is regarded safe for use for lactating mothers.

\section{Isotretinoin}

Isotretinoin is a retinoic acid derivative mostly used in the treatment of nodulocystic acne. It acts on all four etiopathogeneses of acne vulgaris and is capable of prolonged remission and the curing of up to $80 \%$ of patients. It is an FDA-approved treatment for severe recalcitrant acne, which is also useful in moderately treatment-resistant acne, and relapses quickly after the discontinuation of oral antibiotic therapy. Some of the patients using isotretinoin may aggravate their acne after three to six weeks, which may be due to sebaceous gland apoptosis; thus, a low dose of $0.2 \mathrm{mg} / \mathrm{kg} / \mathrm{day}$ may cancel these effects. Low dose isotretinoin (0.2$0.4 \mathrm{mg} / \mathrm{kg} /$ day) have similar effectiveness and fewer side effects [30]. It is generally initiated at $0.5 \mathrm{mg} / \mathrm{kg} /$ day and titrated to $1 \mathrm{mg} / \mathrm{kg} / \mathrm{day}$ as tolerated [31]. A study stated that, as long as treatment with isotretinoin was continued for more than two months after the acne had completely resolved, a cumulative dose or daily dose does not influence acne relapse [32].

\section{Safety Level}

Isotretinoin is the most effective treatment available for acne, but given the ubiquitous distribution of retinoic acid receptors, it almost always causes side effects resembling hypervitaminosis A syndrome. A systematic review shows that its side effects are mostly dermatological, such as skin and mucosal dryness, cheilitis, and peeling of the skin [33]. These are dose-dependent and are also signs of effective drug absorption. Oral isotretinoin may cause spontaneous 
abortion and life-threatening congenital malformations. To reduce teratogenicity, counseling, informed consent, a strict negative pregnancy test, and follow-up should be performed. Regarding mental health, a study published in 1983 asserted a relation between isotretinoin and depressive symptoms. Afterward, numerous articles have triggered controversy around this issue. Most studies have found no association between oral isotretinoin and depression. However, a 2017 metaanalysis revealed that oral isotretinoin produced a significant decrease in depression scales [34]. Oral isotretinoin may cause liver function disarrangements such as increased triglycerides, increased liver transaminases, increased low-density lipoproteins, and decreased high-density lipoproteins, but these side effects are usually short-lived and reversible without withdrawing the medication. Oral isotretinoin is the pregnancy $\mathrm{X}$ category and is not determined yet for nursing.

\section{Emerging Therapies}

Stearoyl coenzyme A desaturase 1 (SCD1) is an enzyme mainly responsible for the synthesis of monounsaturated fatty acids in the sebaceous glands of the skin. The topical application of SCDl inhibitors has shown the potential to reduce the synthesis of monounsaturated fatty acids and the number of sebaceous glands in mouse skin. Several clinical trials of topical formulations of SCDl inhibitors and their potential topical and systemic side effects are ongoing [35].

Nitric oxide-releasing nanoparticles have shown a promising role in preventing C. acnes-induced inflammation. Different investigations are ongoing due to their potential to suppress the release of multiple cytokines from human monocytes and keratinocytes and to prevent C. acnes-induced inflammation [36]. A recent open-label pilot study investigating novel nitric oxide-producing gels showed a decrease in comedones and papules by $50 \%$ in acne lesions [37].

Immunity induction therapy antibodies against the Christie-Atkins-Munch-Peterson (CAMP) factor show cytotoxicity against $C$. acnes and inhibit $C$. acnes growth and the production of murine MIP-2 [38]. Investigations of vaccines produced by Staphylococcus capitis E12 to prevent C. acnes overgrowth and destroy overgrown C. acnes are ongoing [39]. Different monoclonal antibodies blocking cytokines to suppress inflammation are needed as killed C. acnes induces inflammatory cytokines [40].

\section{Light and Laser Therapy}

Light and laser therapies have had their application broadened in the treatment of active acne. Laser therapy is advantageous as it is an in-office treatment and provides no systemic side effects. Red light, blue light, or combined red-blue light leads to the photo-excitation of endogenous bacterial porphyrins, singlet oxygen production, and subsequent C. acnes destruction [41].

Photodynamic therapy has shown the most consistent improvement in acne. A randomized control trial reported 5-aminolevulinic acid-PDT (ALA-PDT) followed by adapalene or oral doxycycline showed a greater reduction of inflammation and the total lesion count in the PDT group at twelve weeks [42]. Intense pulsed light (IPL) is able to destroy C. acnes and lead to the thermolysis of vessels supplying the sebaceous glands. As a result, it decreases sebum production. A study done on ten patients with mild to moderate acne with IPL using a dual-band "notch" acne filter showed that overall lesion clearance was substantial after four weeks of follow-up [43]. However, in recent research comparing Nd: YAG and IPL, a greater reduction of acne lesions, especially of noninflammatory lesions, was seen with Nd: YAG laser [44]. Pulsed dye laser (585-595 nm) uses an organic dye as the laser solution and targets oxyhemoglobin, which causes the heating and photothermolysis of vessels supplying inflammatory acne lesions. A comparison of 585 pulsed dye lasers alone or in combination with Nd: YAG laser showed that inflammatory acne lesions were significantly reduced (82.5-83.5\%) [45].

\section{Fire Needle Therapy}

Fire needle therapy is a form of acupuncture, which exhibits both conventional acupuncture and moxibustion. It is used in traditional Chinese medicine for numerous skin diseases, mainly for the clinical treatment of moderate to severe acne, especially in cystic cases. Fire needle therapy is included in the Chinese Acne Treatment Guidelines [46]. After routine disinfection, an acupuncture needle of $0.15 \mathrm{~mm}$ is heated with an alcohol lamp and pricked in the center of the pustule or cyst. The second or third acupuncture needle is heated until red hot and quickly and accurately pricked into the lesion. A recent study has shown that it is able to reduce the release of inflammatory factors [47], promote circulation, promote metabolism, and restore damaged tissue, 
leading to less scarring [48]. A systematic review and meta-analysis done in 2019 indicated fire needle as a monotherapy or combined with different Chinese and Western medicines, which is equally effective as other conventional modalities of acne treatment [49]. There are no recorded side effects mentioned in the review. Given increasing antibiotic resistance and different undesirable side effects, the currently recommended treatment for acne needs to be upgraded, and alternative modalities should be considered.

\section{CONCLUSION}

Although multiple guidelines recommend limiting the use and decreasing antibiotic resistance, antibiotics are frequently used agents for moderate to severe acne. Topical alternatives, spironolactone, isotretinoin, combined oral contraceptives, and fire needles are all effective therapeutic alternatives. Consideration should be given to these options to improve antibiotic stewardship and proper treatment outcomes for the patient.

\section{Ethics Statement}

The medical ethics committee of the Affiliated Hospital of Inner Mongolia University for the Nationalities approved this study. No potentially identifiable images or data are included in this article.

\section{Author Contributions}

Kiran Sanjel and Xue Mei Zhang contributed to the conception of the study, design and data acquisition, and interpretation, and drafted the article. All authors critically revised and approved the final version.

\section{Statement of Human and Animal Rights}

All the procedures followed were in accordance with the ethical standards of the responsible committee on human experimentation (institutional and national) and with the 2008 revision of the Declaration of Helsinki of 1975.

\section{Statement of Informed Consent}

Informed consent for participation in this study was obtained from all patients.

\section{REFERENCES}

1. Walsh TR, Efthimiou J, Dreno B. Systematic review of antibiotic resistance in acne: An increasing topical and oral threat. Lancet
Infect Dis. 2016;16:e23-e33.

2. Levy RM, Huang EY, Roling D, Leyden JJ, Margolis DJ. Effect of antibiotics on the oropharyngeal flora in patients with acne. Arch Dermatol. 2003;139:467-71.

3. Margolis DJ, Fanelli M, Kupperman E, Papadopoulos M, Metlay JP, Xie SX, et al. Association of pharyngitis with oral antibiotic use for the treatment of acne: A cross-sectional and prospective cohort study. Arch Dermatol. 2012;148:326-32.

4. Margolis DJ, Fanelli M, Hoffstad O, Lewis JD. Potential association between the oral tetracycline class of antimicrobials used to treat acne and inflammatory bowel disease. Am J Gastroenterol. 2010;105:2610-16.

5. Cao Y, Wu K, Mehta R, Drew DA, Song M, Lochhead P, et al. Long-term use of antibiotics and risk of colorectal adenoma. Gut. 2018;67:672-8.

6. Velicer CM, Heckbert SR, Lampe JW, Potter JD, Robertson CA, Taplin SH. Antibiotic use concerning the risk of breast cancer. JAMA. 2004;291:827-35.

7. Kircik LH. The role of benzoyl peroxide in the new treatment paradigm for acne. J Drugs Dermatol. 2013;12:s73-6.

8. Yang Z, Zhang Y, Lazic Mosler E, Hu J, Li H, Zhang Y, et al. Topical benzoyl peroxide for acne. Cochrane Database Syst Rev. 2020;3:CD011154.

9. Kong YL, Tey HL. Treatment of acne vulgaris during pregnancy and lactation. Drugs. 2013;73:779-87.

10. Leyden J, Stein-Gold L, Weiss J. Why topical retinoids are mainstay of therapy for acne. Dermatol Ther (Heidelb). 2017;7:293-304.

11. Han G, Armstrong AW, Desai SR, Guenin E. Novel tretinoin $0.05 \%$ lotion for the once-daily treatment of moderate-to-severe acne vulgaris in an asian population. J Drugs Dermatol. 2019;18:910-6.

12. Tan J, Thiboutot D, Popp G, Gooderham M, Lynde C, Del Rosso J, et al. Randomized phase 3 evaluation of trifarotene $50 \mu \mathrm{g} / \mathrm{g}$ cream treatment of moderate facial and truncal acne. J Am Acad Dermatol. 2019;80:1691-9.

13. Butler DC, Heller MM, Murase JE. Safety of dermatologic medications in pregnancy and lactation: Part II Lactation. J Am Acad Dermatol. 2014;70(3):417.e1-427

14. Cassiopea Receives FDA Approval for Winlevi (clascoterone cream $1 \%$ ), first in class in Topical acne treatment targeting Androgen Receptor". Cassiopea (press release). 2020.

15. Kircik LH. What's new in the management of acne vulgaris. Cutis. 2019;104:48-52.

16. Barbieri JS. A new class of topical acne treatment addressing the hormonal pathogenesis of acne. JAMA Dermatol. 2020;156:619-20.

17. Kalabalik-Hoganson J, Frey KM, Ozdener-Poyraz AE, Slugocki M. Clascoterone: A novel topical androgen receptor inhibitor for the treatment of acne. Ann Pharmacother. 2021;3:1060028021992053.

18. Hebert A, Thiboutot D, Stein Gold L, Cartwright M, Gerloni M, Fragasso E, Mazzetti A. Efficacy and safety of topical clascoterone cream, $1 \%$, for treatment in patients with facial acne: Two phase 3 randomized clinical trials. JAMA Dermatol. 2020;156:621-30.

19. Layton AM, Eady EA, Whitehouse H, Del Rosso JQ, Fedorowicz Z, van Zuuren EJ. Oral spironolactone for acne vulgaris in adult females: A hybrid systematic review. Am J Clin Dermatol. 2017;18:169-91.

20. Barbieri JS, James WD, Margolis DJ. Trends in prescribing behavior of systemic agents used in the treatment of acne among dermatologists and nondermatologists: A retrospective analysis, 2004-2013. J Am Acad Dermatol. 2017;77:456463.e4.

21. Roberts EE, Nowsheen S, Davis DMR, Hand JL, Tollefson MM, Wetter DA. Use of spironolactone to treat acne in adolescent females. Pediatr Dermatol. 2021;38:72-6.

22. Park JH, Bienenfeld A, Orlow SJ, Nagler AR. The use of hormonal antiandrogen therapy in female patients with acne: A 10-year retrospective study. Am J Clin Dermatol. 2018;19:449-55

23. Poinas A, Lemoigne M, Le Naour S, Nguyen JM, Schirr-Bonnans S, Riche VP, et al. FASCE, the benefit of spironolactone for treating 
acne in women: study protocol for a randomized double-blind trial. Trials. 2020;21:571.

24. Plovanich M, Weng QY, Mostaghimi A. Low usefulness of potassium monitoring among healthy young women taking spironolactone for acne. JAMA Dermatol. 2015;151:941-4.

25. Food and Drug Administration. Aldactone (spironolactone) tablets. Physician Labeling. Accessed November 9, 2017.

26. Thorneycroft IH, Stanczyk FZ, Bradshaw KD, Ballagh SA, Nichols M, Weber ME. Effect of low-dose oral contraceptives on androgenic markers and acne. Contraception. 1999;60:255-62.

27. Nast A, Dréno B, Bettoli V, Degitz K, Erdmann R, Finlay AY, et al. European evidence-based (S3) guidelines for the treatment of acne. J Eur Acad Dermatol Venereol. 2012;1:1-29.

28. Lortscher D, Admani S, Satur N, Eichenfield LF. Hormonal contraceptives and acne: a retrospective analysis of 2147 patients. J Drugs Dermatol. 2016;15:670-4.

29. Reid RL. Oral contraceptives and venous thromboembolism: Pill scares and public health. J Obstet Gynaecol Can JOGC. 2011;33:1150-5.

30. Faghihi G, Mokhtari F, Fard NM, Motamedi N, Hosseini SM. comparing the efficacy of low dose and conventional dose of oral isotretinoin in treatment of moderate and severe acne vulgaris. J Res Pharm Pract. 2017;6:233-8.

31. Zaenglein AL, Pathy AL, Schlosser BJ, Alikhan A, Baldwin HE, Berson DS, et al. Guidelines of care for the management of acne vulgaris. J Am Acad Dermatol. 2016;74:945-73.e33.

32. Rademaker M. Making sense of the effects of the cumulative dose of isotretinoin in acne vulgaris. Int J Dermatol. 2016;55:518-23.

33. Vallerand IA, Lewinson RT, Farris MS, Sibley CD, Ramien ML, Bulloch AGM, Patten SB. Efficacy and adverse events of oral isotretinoin for acne: A systematic review. Br J Dermatol. 2018;178:76-85.

34. Huang Y-C, Cheng Y-C. Isotretinoin treatment for acne and risk of depression: A systematic review and meta-analysis. J Am Acad Dermatol. 2017;76:1068-76.e9.

35. Zouboulis CC, Dessinioti C, Tsatsou F, Gollnick HPM. Antiacne drugs in phase 1 and 2 clinical trials. Expert Opin Investig Drugs. 2017;26:813-23.

36. Qin M, Landriscina A, Rosen JM, Wei G, Kao S, Olcott W, et al. nitric oxide-releasing nanoparticles prevent propionibacterium acnes-induced inflammation by both clearing the organism and inhibiting microbial stimulation of the innate immune response. J Invest Dermatol. 2015;135:2723-31.

37. Settelmeier S, Rassaf T, Hendgen-Cotta UB, Stoffels I. nitric oxide generating formulation as an innovative approach to topical skin care: An open-label pilot study. Cosmetics. 2021;8:16.
38. Wang Y, Hata TR, Tong YL, Kao MS, Zouboulis CC, Gallo RL, et al. The anti-inflammatory activities of propionibacterium acnes camp factor-targeted acne vaccines. J Invest Dermatol. 2018;138:2355-64.

39. O’Neill AM, Nakatsuji T, Hayachi A, Williams MR, Mills RH, Gonzalez DJ, et al. Identification of a human skin commensal bacterium that selectively kills cutibacterium acnes. J Invest Dermatol. 2020;140:1619-28.

40. Lyte P, Sur R, Nigam A, Southall MD. Heat-killed Propionibacterium acnes is capable of inducing inflammatory responses in skin. Exp Dermatol. 2009;18:1070-2.

41. Nestor MS, Swenson N, Macri A. Physical modalities (devices) in the management of acne. Dermatol Clin. 2016;34:215223.

42. Nicklas C, Rubio R, Cárdenas C, Hasson A. Comparison of efficacy of aminolaevulinic acid photodynamic therapy vs. adapalene gel plus oral doxycycline for treatment of moderate acne vulgaris- $A$ simple, blind, randomized, and controlled trial. Photodermatol Photoimmunol Photomed. 2019;35:3-10.

43. Knight JM. Combined 400-600nm and 800-1200nm intense pulsed phototherapy of facial acne vulgaris. J Drugs Dermatol. 2019;18:1116-22.

44. Monib KME, Hussein MS. Nd: YAG laser vs IPL in inflammatory and noninflammatory acne lesion treatment. J Cosmet Dermatol. 2020;19:2325-32

45. Salah El Din MM, Samy NA, Salem AE. Comparison of pulsed dye laser versus combined pulsed dye laser and Nd: YAG laser in the treatment of inflammatory acne vulgaris. J Cosmet Laser Ther. 2017;19:149-59.

46. Ju Qiang. Chinese acne treatment guidelines(2019 Revision). J Clin Dermatol. 2019;49:584.

47. Zhou Z, Li Y. Effect of fire on expression of IL-1beta and caspase -3 protein in rat model of spinal cord injury. Shanghai $J$ Acupunct Moxibust. 2010;29:318-21.

48. Rui Z. Clinical observation of isotretinoin combined with fire needle therapy in treatment of grade IV acne. J Pract Chin Med. 2016,32:278

49. Xing M, Yan X, Sun X, Wang S, Zhou M, Zhu B, et al. Fire needle therapy for moderate-severe acne: A PRISMA systematic review and meta-analysis of randomized controlled trials. Complement Ther Med. 2019;44:253-60.

Copyright by Kiran Sanjel, et al. This is an open access article distributed under the terms of the Creative Commons Attribution License, which permits unrestricted use, distribution, and reproduction in any medium, provided the original author and source are credited.

Source of Support: Nil, Conflict of Interest: None declared. 